\title{
Wilms' tumour and renal dysplasia: an hypothesis
}

\author{
HB MARSDEN, W LAWLER* \\ From the Royal Manchester Children's Hospital, Manchester M27 1HA and the *Department of Pathology, \\ University of Manchester, Oxford Road, Manchester M13 9PT
}

SUMMARY The incidence of renal dysplasia in a series of Wilms' tumours is presented. The distribution of such lesions is discussed, together with their course of development and regression. The kidney is regarded as a particularly suitable organ for studying the relation between dysplasia and neoplasia. A schema is suggested for this association with regard to Wilms' tumour.

Paediatric oncology offers the opportunity to study the interrelations between normal embryonic development, dysplasia and neoplasia. The kidney is a particularly suitable organ for such study as there is a clear relation between renal dysplasia and Wilms' tumour.' The dysplastic features have been emphasised' under the general term "the nephroblastomatosis complex," with "nodular renal blastema" and "sclerosing metanephric hamartoma" as main subgroups and further subdivision of the latter into three types. Associated changes of glomerular immaturity and sclerosis and of focal cortical cysts ${ }^{1}$ are less clearly dysplastic features allied to the development of a Wilms' tumour and may even be secondary to neoplasia within the kidney. Because the term "nephroblastomatosis" has been applied specifically to multifocal and widespread epithelial dysplastic lesions ${ }^{2}$ and because in our experience mesodermal in addition to epithelial hamartomata may be rarely encountered, it has been avoided and "renal dysplasia" used.

Dysplastic lesions consist of metanephric tissue which is predominantly tubular but may also show primitive glomerular formation. The tubules are different from those seen in Wilms' tumours and consist of small rounded or polygonal hyperchromatic cells rather than the columnar cells which are encountered in nephroblastomas. There is considerable variation in the extent of dysplastic change, varying between widespread confluent lesions and involvement of a single tubule. The small size of the minimal lesions precludes adequate assessment of the true incidence of dysplasia. Fibrosis is commonly encountered in dysplastic lesions and complete sclerosis with loss of tubular elements appears to be the natural course of evolution. It is therefore not possible to exclude previous dysplastic change

Accepted for publication 28 January 1982 when isolated areas of fibrosis are seen and this further compounds the difficulty of assessing the true incidence of dysplasia. Occasionally, bands of smooth muscle rather than epithelial differentiation are encountered and are difficult to interpret; it is possible that these may represent mesenchymal elements in renal dysplasia. (Fig. 1).

The location of the dysplastic lesions relative to the Wilms' tumour has important implications concerning the relation between dysplasia and neoplasia (see Discussion). Three main sites for dysplasia are seen:

1 The immediate subcapsular region of the kidney, this being the most common (Fig. 2).

2 The tumour-kidney margin. Compression atrophy of pre-existing normally developed kidney makes assessment of dysplasia difficult at this site (Figs. 3 and 4 ).

3 Within the tumour itself. This is a particular feature of more highly differentiated epithelial tumours (Fig. 5) and is easily recognised in fibrous septa (Fig. 6). Occasionally, there may be extension along tissue septa to the periphery and the tumour-kidney junction. (Fig. 3).

In a recent histopathological analysis by the present authors of 133 consecutive unilateral Wilms' tumours, nephroblastic dysplasia was found in 24 (18.0\%), 22 epithelial and two mesodermal in type.

In this series, congenital anomalies were seen as follows: naevi and haemangiomata 7; definite hemihypertrophy 4 ; pyloric stenosis 3 ; duplex kidney 2 ; Beckwith-Wiedemann syndrome 2 ; and one each of horseshoe kidney, umbilical hernia, urethral valves, renal cysts, Hirschprung's disease and multiple skeletal abnormalities. Two cases of congenital heart disease, one Ollier's disease and one Hirschprung's disease were noted in siblings. There was one case of congenital heart disease and one of heterochromia iridis in parents. In one patient, renal 


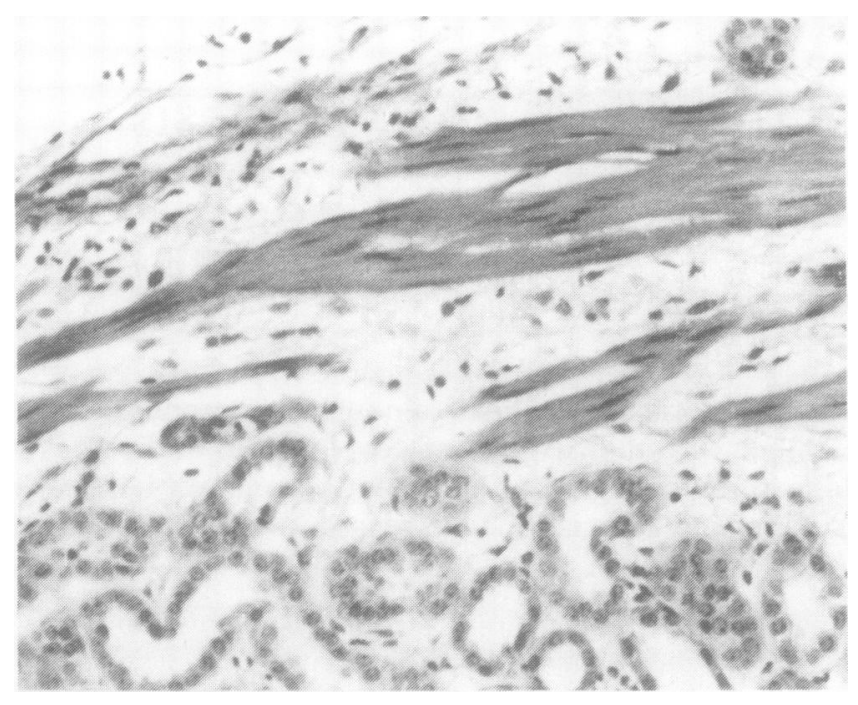

Fig. 1 Smooth muscle bundle in the subcapsular region in a kidney which contained a Wilms tumour. There is loose surrounding connective tissue but no blastema. Haematoxylin and eosin $\times 150$.

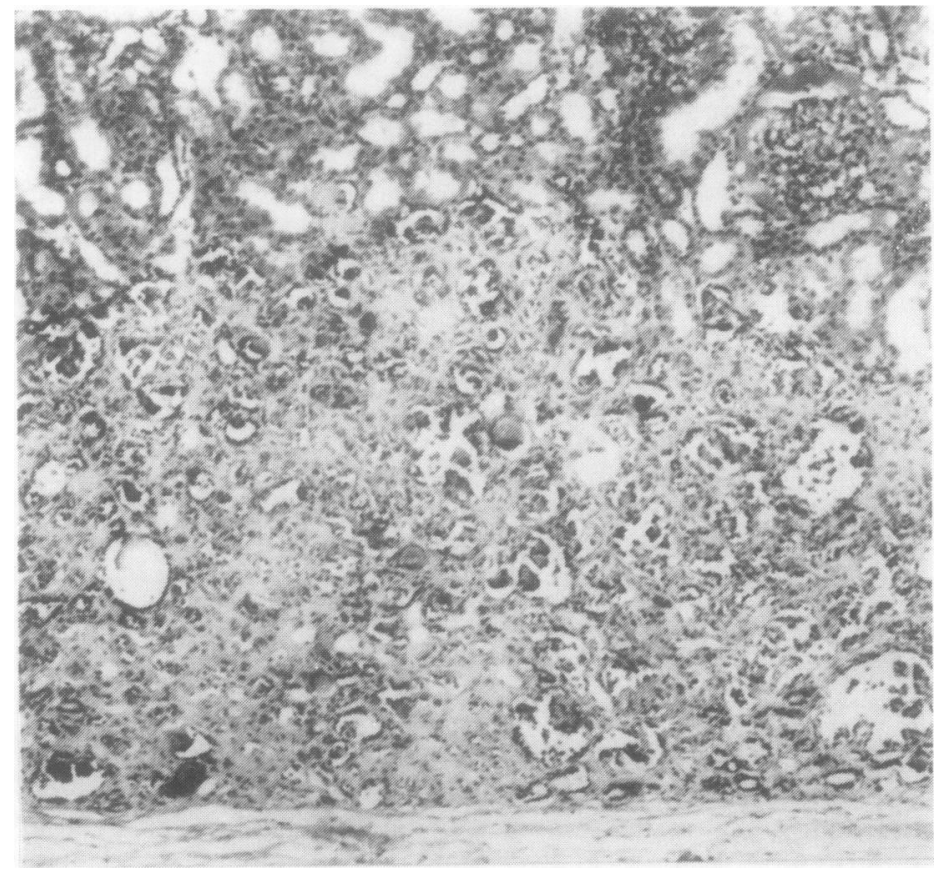

Fig. 2 Subcapsular focus of sclerosing renal nephroblastic dysplasia. Haematoxylin and eosin $\times 55$. carcinomata developed in two great grandparents (one paternal, one maternal). The only association between nephroblastic dysplasia and congenital anomalies in this particular series was one of the two patients with Beckwith-Wiedemann syndrome who also had renal cysts and haemangiomata.

\section{Discussion}

In an attempt to correlate the various observations on dysplasia and childhood renal neoplasia, a suggested hypothesis is summarised in the accompanying diagram (Fig. 7). 


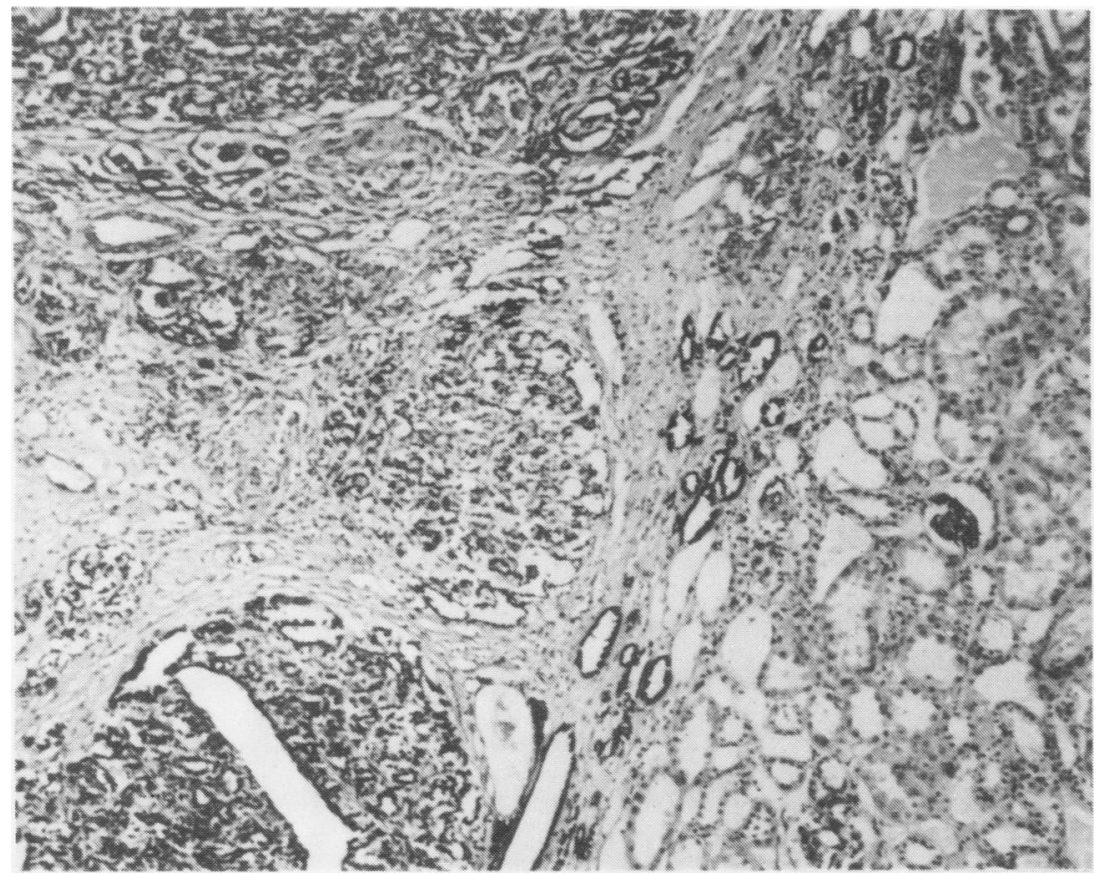

Fig. 3 Nephroblastic dysplasia at the tumour-kidney junction with extension into the tumour itself. Haematoxylin and eosin $\times 55$.

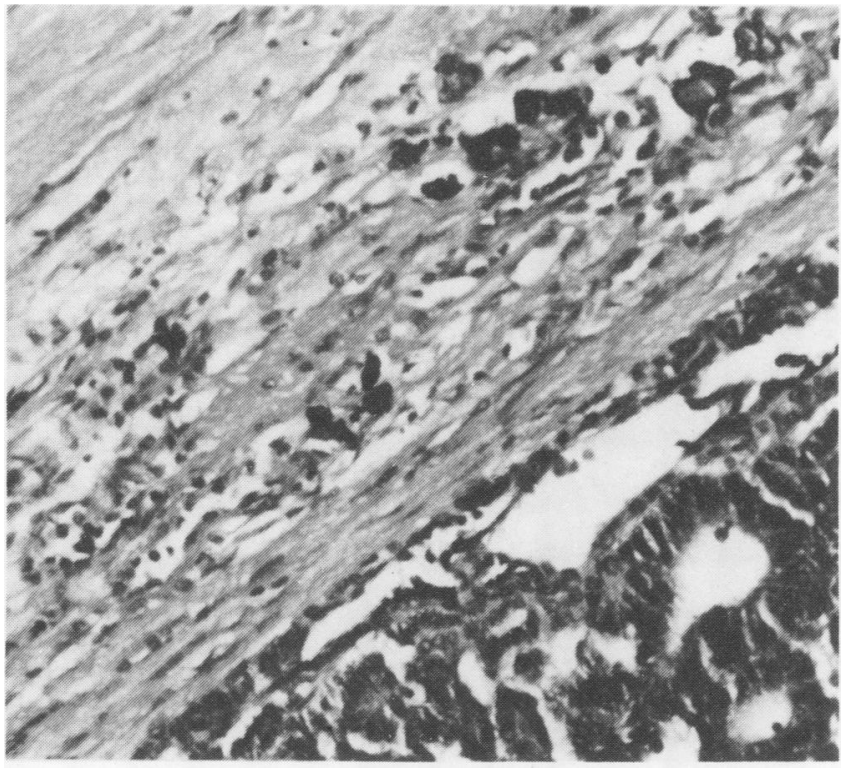

Fig. 4 Sclerosing nephroblastic dysplasia with focal calcification at the tumour-kidney junction. Haematoxylin and eosin $\times 150$.

During normal kidney development, pluripotential metanephrogenic blastema becomes committed to form purely renal tissue. Although histological appearances indicate that Wilms' tumour is normally derived from committed blastema, the occasional finding of heterologous elements (cartilage, fat, bone etc) suggests that the neoplasm may arise from uncommitted metanephrogenic blastema. The metanephric composition of the dysplastic lesions points to an origin from committed blastema and the occasional finding of smooth muscle would not refute this possibility. 


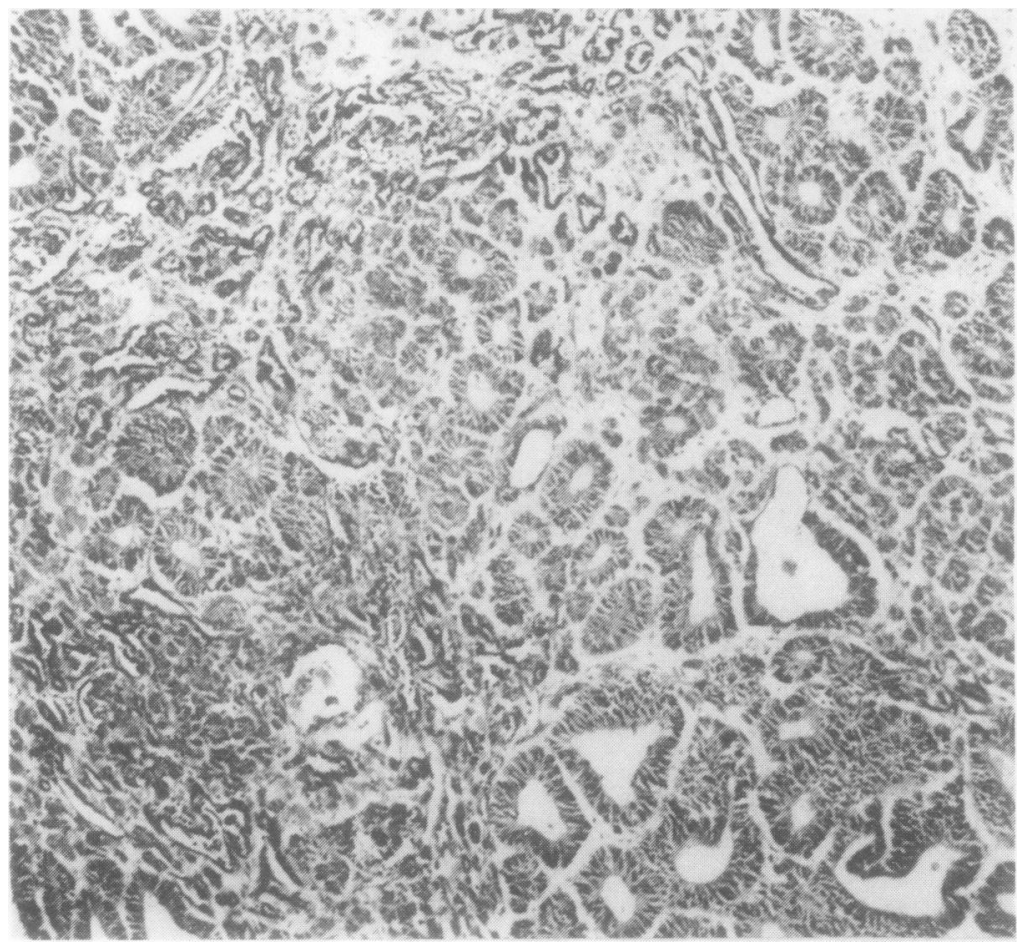

Fig. 5 Nephroblastic dysplasia within a highly differentiated epithelial Wilms' tumour.

Haematoxylin and eosin $\times 55$.

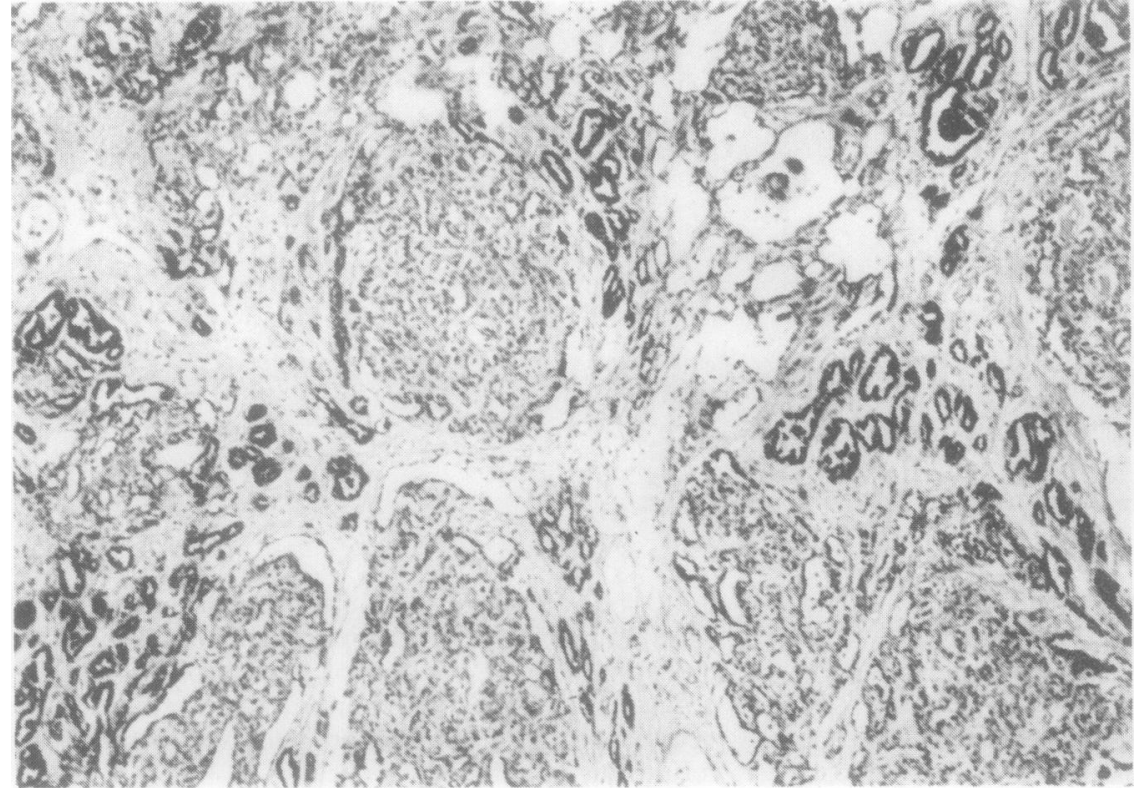

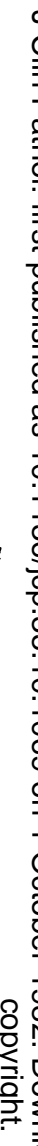

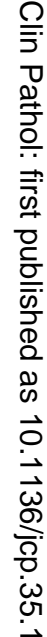

0
0
0
0
0
0
0
0
0
0
0
0

음

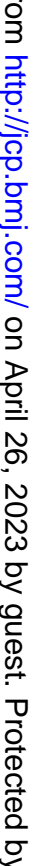

Fig. 6 Nephroblastic dysplasia in fibrous septa within a Wilms' tumour. Haematoxylin and eosin $\times 55$. 


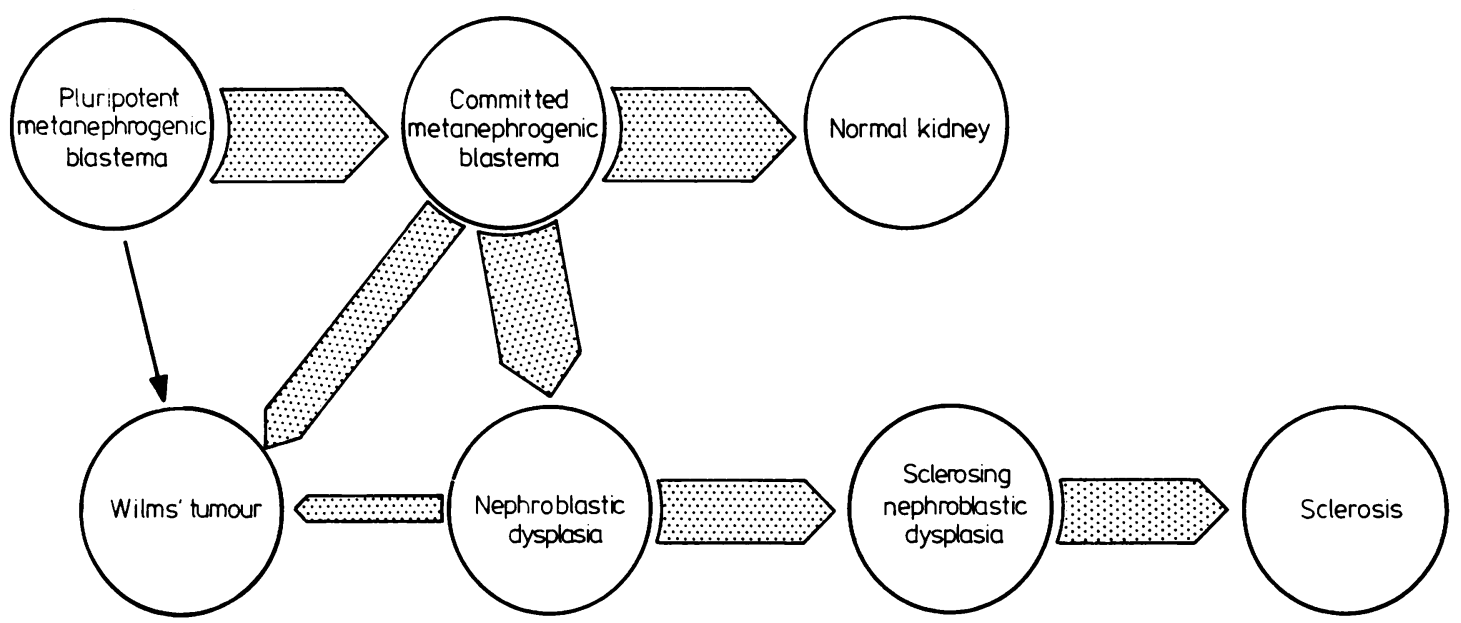

Fig. 7 Suggested relations between normal, dysplastic and neoplastic renal development. The relative width of the arrows indicates the probability of the course of events although the arrows representing normal development have of necessity had to be reduced in size.

The relatively high incidence of tumours with striated muscle as the only heterologous element suggests that, although not shown in our Fig. 7, neoplasms may arise from an intermediate stage between pluripotential and committed metanephrogenic blastema.

The close anatomical association between dysplasia and neoplasia (see above) raises the possibilities either that the tumour may arise directly from dysplastic foci or that the two are independent but linked aetiologically.

The existence of dysplastic areas within differentiated rather than undifferentiated tumours may be explained in three ways. Firstly, the development of neoplasia from dysplasia may lead to the formation of a highly tubular tumour; secondly, differentiated rather than undifferentiated tumours and dysplasia may have the same origin; thirdly, more aggressive blastemal neoplasms may destroy pre-existing dysplastic elements. However, it is clear that well differentiated tumours and dysplasia may coexist without apparent damage to the latter.

The $18 \%$ incidence of nephroblastic dysplasia in our recently analysed series is rather lower than the $33.3 \%$ of Bove and McAdams, ${ }^{1}$ but bilateral cases were excluded from our series, and these authors ${ }^{1}$ emphasise the high association between bilateral tumours and metanephrogenic abnormalities. Knudson and Strong ${ }^{3}$ concluded that bilateral tumours are more likely to be familial, that familial tumours result from two mutations, one germinal and one somatic, and that sporadic tumours result from two somatic mutations. However, nephroblastic dysplasia is a feature of both unilateral and bilat- eral Wilms' tumours. Although some family data is available from the patients in our series, the minimal association between nephroblastic dysplasia and congenital anomalies together with the absence of bilateral tumours and any family history of nephroblastoma preclude significant genetic assessment.

Wilms' tumours are neoplasms associated with the embryological development of the kidney, and are typically located deeply in the renal substance: it is even possible, considering their well-defined margins, that they are initiated prior to organoid renal development. The finding of subcapsular dysplastic foci with a deep-seated neoplasm indicates that the formation of such foci is not confined to the initial period of oncogenesis but may occur subsequently.

\section{References}

' Bove KE, McAdams AJ. The nephroblastomatosis complex and its relationship to Wilms' tumor: a clinicopathologic treatise. Perspect Pediatr Pathol 1976;3:185-223.

${ }^{2}$ Hou LT, Holman RL. Bilateral nephroblastomatosis in a premature infant. J Pathol Bacteriol 1961;82:249-55.

${ }^{3}$ Knudson AG, Strong LC. Mutation and cancer: a model for Wilms' tumour of the kidney. $J$ Natl Cancer Inst 1972;48:313-24.

Requests for reprints to: Dr HB Marsden, Consultant Pathologist, Royal Manchester Children's Hospital, Manchester M27 1HA, England. 